\title{
Building an Optimal WSD Ensemble Using Per-Word Selection of Best System
}

\author{
Harri M.T. Saarikoski ${ }^{1}$ and Steve Legrand ${ }^{2}$ \\ ${ }^{1}$ KIT Language Technology Doctorate School, Helsinki University, Finland \\ Harri.Saarikoski@helsinki.fi \\ ${ }^{2}$ Department of Computer Science, University of Jyväskylä, Finland \\ stelegra@cc.jyu.fi
}

\begin{abstract}
In Senseval workshops for evaluating WSD systems [1,4,9], no one system or system type (classifier algorithm, type of system ensemble, extracted feature set, lexical knowledge source etc.) has been discovered that resolves all ambiguous words into their senses in a superior way. This paper presents a novel method for selecting the best system for target word based on readily available word features (number of senses, average amount of training per sense, dominant sense ratio). Applied to Senseval-3 and Senseval-2 English lexical sample state-of-art systems, a net gain of approximately $2.5-5.0 \%$ (respectively) in average precision per word over the best base system is achieved. The method can be applied to any base system or target word in any language.
\end{abstract}

\section{Introduction}

Based on recent evaluation of WSD systems, progress in disambiguation methods have reached a standstill. The 15 best systems in Senseval-3 English sample task ended up within $2 \%$ of each other [10] while in Senseval-2 the number of systems within that range was only five [1]. Numerous methods of disambiguation have been tried out in Senseval evaluations. For instance, most classifiers found effective in data mining experiments have been tried out: in Senseval-3 for example there were experiments with support vector machines (IRST-kernel, nusels), Naive Bayes (CLaC1, all htsa systems), Neural Networks (MC-WSD, UJAEN) and Maximum Entropy algorithms (HKUST-me, CLaC2) [10]. Multi-classifier experiments have also been very popular $[3,19,12,17]$ : in Senseval-3 evaluation, classifier ensembles were as popular as single-classifier systems (e.g. SyntaLex, NRC, HKUST-all and BCU systems, and Duluth-ELSS) [10].

The first conclusion from these experiments has been that different disambiguation methods result in different performance results. A second conclusion is that there is a 'word bias', i.e. each word poses a different set of learning problems. To solve these biases, all we need is an exact definition of the type of system that is best equipped to handle a particular target word. [18] showed that word grain, amount of training and most frequent (dominant) sense bias in training data are factors that have a profound influence on system performance. For instance, disambiguating a hard word (40 
senses, average of 4 training examples per sense out of which dominant sense gets $25 \%$ ) is a different type of learning task than disambiguating an easy word (2-sense word with 40 examples at $80 \%$ dominant share). Since classifiers have different solutions to deal with the different learning tasks, it is reasonable to assume that system strengths tend to follow changes (drops and rises) in these three word factors. We further propose that system strength is focused on a particular region of this 'word space' (see Figures 1 and 2), which allows effective predictors of best system per word to be built.

This paper presents a novel method using the three word features that fairly accurately predicts the strong regions of given base systems. To our knowledge, only one such per-word ensemble using word features as system selection criterion has been implemented [11] where they selected the system according to target word partof speech. Despite the fact that the two-system ensemble ended up at the bottom of the Senseval-2 evaluation (20\% off the state of the art), it still achieved three wordwins, which indicates the viability of the per-word selection method in general.

In section 2, we present the machine-learning tools we used for predictions. In section 3, we define the three word-based factors and the predictors built on them. In section 4 , we present the disambiguation method based on those predictors, and in section 5, we test the method in practice on two different datasets. Sections 6 and 7 discuss and conclude on the findings.

\section{MOA-SOM Toolkit}

Study of disambiguation systems lacks a diagnostic tool that could be used to metalearn the effects of these factors. As a result, the following types of questions are largely unanswered: Which are the words where a system is at its strongest? What type of ensembles of systems achieve optimum performance for give target word?

We are developing a meta-classifier (MOA-SOM, 'mother-of-all-self-organizingmaps') to handle such learning tasks. The tool clusters publicly available WSD system scores $[10,1,4]$ stored in database [13] based on features defining the systems (e.g. classifier algorithm, feature sets) and target words (e.g. PoS, training, word grain) by calculating the amount of correlation between systems and words. The output from MOA-SOM is the optimal classifier, feature and configuration for that target word. The feature matrix can be fed to SOM using either system names as labels and words as data points or vice versa. The SOM used is based on hierarchically clustering DGSOT [7] which was found useful in earlier WSD experiments [6]. For these tests we additionally employed the machine-learning algorithms implemented in Weka toolkit [16] for predictors.

In the next section, we present the three factors in more detail and how we combined them to build machine-learning predictors of system differences.

\section{Predictor Building}

In this section we present the factors predicting system performance and the predictors using those factors for prediction of system differences. 


\subsection{Factors}

We introduce here the three word-based factors in explaining variations in system performance (Train, Grain, and DomSub). Train is average number of training instances per sense, Grain is the number of senses (as recorded in WordNet / WordSmyth sense repositories used in Senseval evaluations). DomSub is aimed to differentiate between systems that differ in their inherent bias to deal with big vs small dominant (most frequent) sense shares. The formula we used for DomSub is Domsub $=\mathrm{dom}^{2}+\mathrm{sub}^{2}$ where dom and $s u b$ are the shares of dominant and subdominant sense out of all training instances for the current target word ${ }^{1}$. For example, for a word with $80 \%$ / $20 \%$ shares for dominant / subdominant senses, DomSub is $0.8^{2}+0.2^{2}=0.68$.

Next we present the types of predictors we used in our experiments.

\subsection{Predictors}

A few factor formulas emerged as best predictors of system difference predictors. To train the predictors, we used both manual rules and machine-learning algorithms:

(1) Bisections (baseline). To achieve a bisection baseline, we first sort the data according to a selected factor (e.g. T, G, D, T+G+D), then split the data in two and calculate the net gain by each system for each half and average that by dividing it by two. The best weighting scheme we found was the square root of the unweighted sum of normalized values of the three factors: sqrt $(a * T+b * G+c * D)$ where $G$ stands for Grain, $T$ for Train, $D$ for DomSub values of target words and integers $a, b$ and $c$ normalize the weights of the three factors. Note that since this set of predictors is limited to one factor at a time, it cannot express decision rules containing multiple factors which tends to make them less reliable.

(2) Machine-learned models. To predict the best system for words, we trained some of the most efficient learning algorithms implemented in Weka toolkit [16] (Support Vector Machine, Maximum Entropy, Naive Bayes, Decision Trees, Random Forests as well as voting committee, training data bagging and algorithm boosting methods). For training we used the abovementioned word factors both individually and in various permutations (e.g. T-G).

Next we outline the method of using these predictors for system-word pairing.

\section{Method}

In this section, we outline a method for defining and selecting maximally complementary base systems integrated inside a disambiguation algorithm:

1. Base system selection. Run candidate base systems on training words. Investigate their performance at different types of words. Based on their performance at training words, select systems whose strong regions are as large and as distinct as possible using the following criteria:

\footnotetext{
${ }^{1} \mathrm{We}$ consider the increment from the rest of the senses (typically < 0.05$)$ as largely negligible.
} 
biggest gross gain (see Evaluation) from candidate base systems

$\bigcirc$ largest number of training words won by the system

2. Training the predictor. Using the training run data, train the predictors to recognize the best base system using readily available factors (e.g. word grain). Predictor can be constructed by setting decision rules manually, e.g. "use system\#1 (Decision Tree -based) when number of senses (grain) $<5$, system\#2 (Naive Bayes -based) when grain is $>5$ but not when $20<$ train $<$ 25". Alternatively, use a machine-learning algorithm to induce the rules from the training data.

3. Testing. Run selected base systems and the ensemble (according to the best predictor for that ensemble) on test words.

4. Evaluation. Evaluate the ensemble by comparing it to the better of the base systems. Also evaluate the predictor using net gain measure calculated from the following formula:

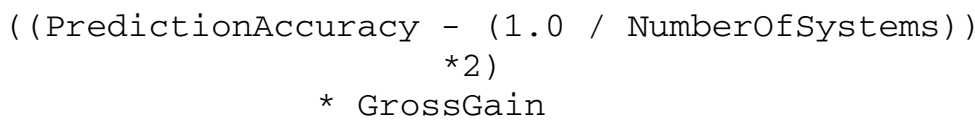

PredictionAccuracy is the number of correct system-for-word predictions out of all test words and NumberOfSystems is the number of classes/systems to predict. GrossGain is a measure of the potential of the base systems when they form an ensemble, resulting from a perfect system-for-word prediction for all test words. It is calculated from all-words average net gain by either base system (e.g. in a test set of two words, if system\#1 wins over system\#2 by $2 \%$ at word\#1 and system\#2 wins over system\#1 by $4 \%$ at word\#2, then gross gain for all test words is $(2+4) / 2=3 \%)$. Net gain is then calculated as follows: in a two-system ensemble with 0.80 prediction accuracy and $8.0 \%$ gross gain, net gain is $((0.80-0.50) * 2)) * 8.0 \%=4.8 \%$. It should be noted that in a two-system prediction task, prediction accuracy of 0.50 results in zero net gain, same as random selection of system.

Next we apply this method to two separate Senseval datasets (four prediction tasks each), using state of the art systems and predictors that proved best in our tests.

\section{Testing}

In this section, we apply the method to two Senseval evaluations.

\subsection{Senseval-2 English Lexical Sample}

System Selection. We trained the predictors with 39 words ${ }^{2}$ and considered all supervised systems as candidates for base systems ${ }^{3}$. We selected the systems based on criteria in Step 1 of the method: looking at wins by best systems in training words, SMU [9] got 10 wins, JHU [17] nine, KUN(LP) [14] got four and CS(224N) [8] three.

\footnotetext{
${ }^{2}$ We discarded words where the wordwinner system's margin over next best system was $<2 \%$.

${ }^{3}$ We ignored low-recall $(<99 \%)$ and low-precision (> 4\% behind best) systems.
} 
Strong region of the latter was almost identical the same as that of JHU, yet smaller, and even though the abovementioned Alicante system [8] scored 3 wordwins, it cannot be used because of its poor overall performance ( $20 \%$ behind top) (criteria 3 ). In Figure 1, we see the Train-Grain regions (most important criteria 1) of the two top 'wordwinners' (SMU and JHU).

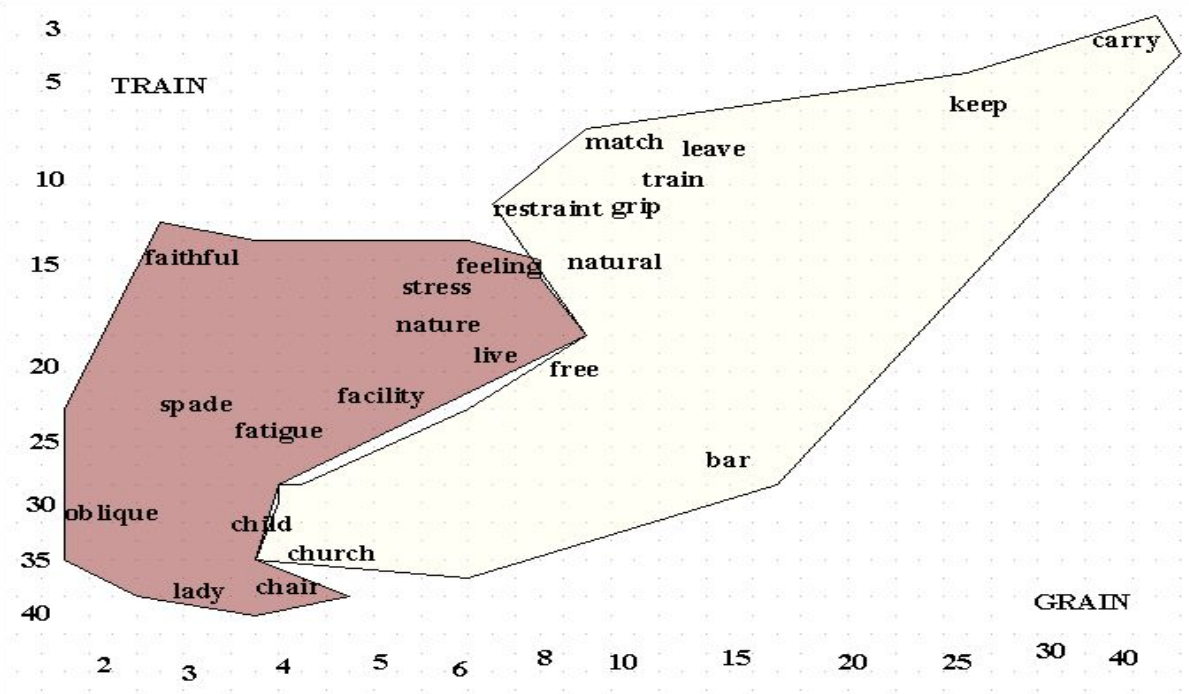

Fig. 1. Strong regions of two Senseval-2 systems in Train-Grain space (sample of training words shown). JHU region can be found on right (mid/high-grain), SMU region on left (lowgrain, mid/high-train).

Table 1. Results from applying the method on selected base systems from Senseval-2

\begin{tabular}{lrcc}
\hline $\begin{array}{l}\text { system pair } \\
\text { (gross gain) }\end{array}$ & $\begin{array}{l}\text { best predictor } \\
\text { (factor/classifier) }\end{array}$ & $\begin{array}{l}\text { prediction } \\
\text { accuracy }\end{array}$ & $\begin{array}{l}\text { net gain } \\
\text { of ensemble }\end{array}$ \\
\hline JHU+SMU (8.0\%) & (1) (T-G) / (T+G) & 0.63 & $2.6 \%$ \\
& (2) SVM * & 0.80 & $4.8 \%$ \\
SMU+KUN (8.4\%) & (1) T+G+D & 0.70 & $3.4 \%$ \\
& (2) SVM & $\mathbf{0 . 8 2}$ & $\mathbf{5 . 0 \%}$ \\
JHU+KUN (5.5\%) & (1) T+G+D & 0.56 & $1.7 \%$ \\
& (2) SVM & 0.75 & $2.8 \%$ \\
JHU+SMU+KUN (9.5\%) & (3) SVM & $0.55^{4}$ & $4.2 \%$ \\
\hline
\end{tabular}

We see from Figure 1 that SMU and JHU populate distinct and large learning regions in Grain-Train space. KUN (not showing) also occupies a large region, focused on high-grain, low-train words such as call and dress and would be located approximately between JHU's strong words keep and leave.

\footnotetext{
${ }^{4}$ Note that having three systems to predict yields a naturally lower prediction accuracy.
} 
Strength of KUN is, however, in the steady quality of its performance with all words, not manifesting any huge drops with any word.

Testing. We tested the model(s) on 19 words and three possible two-system combinations of the three top wordwinning systems (SMU, JHU and KUN) as well as an ensemble of all three systems.

SMU+KUN appears to have the highest gross gain, prediction accuracy and net gain, making it the maximally complementary system pair for this dataset. Furthermore, it seems that 3-system prediction (JHU+SMU+KUN) with more gross gain loses to 2-system predictions in prediction accuracy ending up with a slightly lower net gain.

\subsection{Senseval-3 English Lexical Sample}

System Selection. We trained the predictors again with 39 words and considered 15 top systems and selected the three top wordwinners for candidate base systems: IRSTkernel [15] with 8, htsa3 [2] 4 and nusels [5] with 3 training words won. Let us investigate the strong Train-Grain regions of the two top wordwinners.

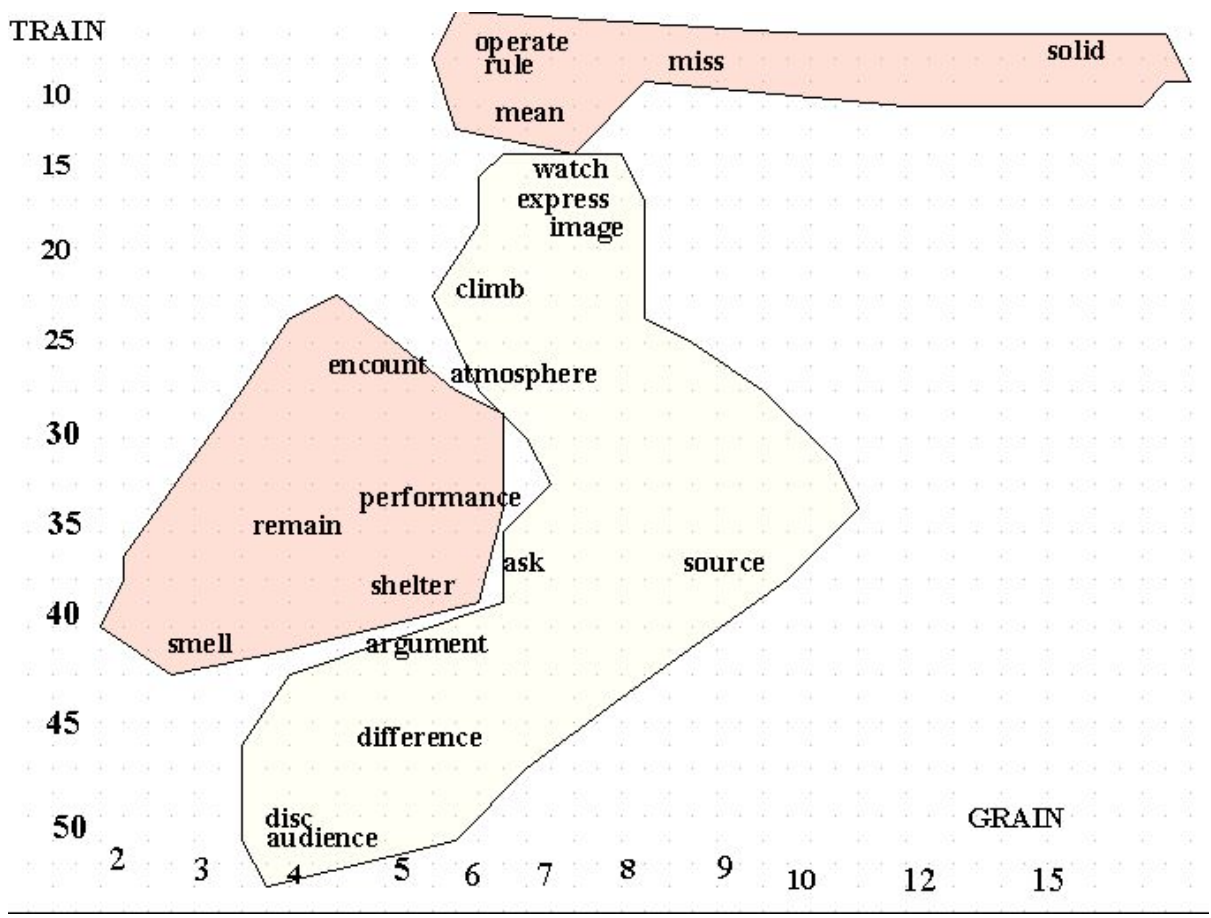

Fig. 2. Strong regions of two Senseval-3 systems in Train-Grain space (sample of training words shown). htsa3 is the lighter shade intact region in the middle, IRST-kernel holds the other two regions, one on left, one on top. 
These two systems (htsa3 and IRST-kernel) complement each other very well. IRST-kernel occupies two regions but since training data contains no words from this area the regions cannot be merged.

Testing. We tested the model on 19 words and three two-system combinations of the three wordwinning systems (htsa3, IRST-kernel and nusels) as well as an ensemble of all three systems.

Table 2. Results from applying the method on selected base systems from Senseval-3

\begin{tabular}{lrcc}
\hline $\begin{array}{l}\text { system pair } \\
\text { (gross gain) }\end{array}$ & $\begin{array}{l}\text { best predictor } \\
\text { (factor/classifier) }\end{array}$ & $\begin{array}{l}\text { prediction } \\
\text { accuracy }\end{array}$ & $\begin{array}{l}\text { net gain } \\
\text { of ensemble }\end{array}$ \\
\hline htsa3+IRST-kernel (4.1\%) & (1) T+G+D & 0.80 & $2.5 \%$ \\
& (2) NaiveBayes & $\mathbf{0 . 8 2}$ & $\mathbf{2 . 7 \%}$ \\
htsa3+nusels (3.6\%) & (1) T+G+D & 0.65 & $1.2 \%$ \\
& (2) DecisionTree & 0.70 & $1.4 \%$ \\
nusels+ IRST-kernel $(\mathbf{4 . 4 \% )}$ & (1) (T-G) / (T+G) & 0.80 & $2.6 \%$ \\
& (2) SVM & 0.80 & $2.6 \%$ \\
htsa3+IRSTk+nusels $(6.1 \%)$ & (2) MaxEnt & 0.55 & $\mathbf{2 . 7 \%}$ \\
\hline
\end{tabular}

Table 2 shows nusels+IK is the maximally complementary system pair in terms of net gain but another system pair (nusels+IRST-kernel) has the higher potential (gross gain). It should also be noted that the more challenging three-system prediction task (htsa3+IRSTk+nusels) produces equally high net gain as htsa3+IRST-kernel pair.

\section{Discussion}

Best predictors turned out to vary according to base system pair, both in terms of learning algorithm and input features. The most reliable learning algorithms turned out to be Support Vector Machines and slightly less consistently Maximum Entropy and Naive Bayes classifiers. Machine-learning models (2) tend to work better than the corresponding bisection baseline (1). The contribution of individual factors to system differentiation seems to depend heavily on the base system pair: combination of factors tended to work better than individual factors but there were (e.g. T+G+D for SMU/JHU pair) but sometimes one factor differentiated better (e.g. DomSub for IRST-kernel/htsa3). These findings lead us to conclude that this system prediction task - just like word sense disambiguation task itself - is in fact a set of tasks dependent on details and the difficulty of the task, and therfore, a customized predictor may need to be developed for given system pair.

\section{Conclusion}

We have presented a novel method for constructing effective WSD system ensembles. Predictors built on word-based factors (Grain, Train, DomSub) seem to yield very good predictions of optimal systems for words. The method was tested with two evaluations: in Senseval-2 the best net gain was 5.0\% (out of maximal $8.4 \%$ ) for 
SMU/JHU pair, while in the more contested Senseval-3 it was $2.7 \%$ (out of $4.1 \%$ ) for three-system ensemble (htsa3/IRST-kernel/nusels). The method is scalable to any ambiguous word and any assortment of base systems and the factors used to build predictors are readily available for all words.

Although most predictors exceed random selection baseline (zero net gain), further work is needed to make the prediction method more accurate and thereby maximize net gain. It should be kept in mind that base systems and their optimal predictors form a pair. Based on a more covering set of factors, we can then learn more reliable predictors, including for more than two or three systems. Particularly we need to account for other factors found influential to system performance: choice of feature sets $[6,18]$ as well as choice of the classifier algorithm as well as the specifics of its sense decision procedure $[2,18,19]$. We also believe it is possible to fabricate two strong, 'opposite' systems that together optimally cover the word space, which probably makes predictions more reliable too. Furthermore, as with any supervised prediction system, providing more training words to a machine learner is likely to improve prediction accuracy (e.g. all the 165 English words and around 100 systems that have participated in the three English Senseval evaluations). Evaluation data from other languages can also be used since our preliminary comparisons with Spanish Senseval-2 data indicate that the same systems (JHU, CS, Duluths, UMCP) that excelled in a particular region in the English evaluation did so in Spanish as well. This phenomenon can be explained by the language-independence of both word factors and WSD systems and suggests that it is possible to build one 'optimal ensemble' that would be as effective for all languages.

\section{Acknowledgments}

Authors would like to express their gratitude to professor Alexander Gelbukh of Instituto Politecnico Nacional, Mexico, for his review of our paper. Work of first author is supported by Department of Translation Studies (Language Technology Doctorate School) of Helsinki University, Finland, and second author by COMAS Doctorate School of Jyväskylä University and Instituto Politecnico Nacional, Mexico.

\section{References}

1. Edmonds, P., and Kilgarriff, A. Introduction to the Special Issue on evaluating word sense disambiguation programs. Journal of Natural Language Engineering 8(4) (2002).

2. Grozea, C. Finding optimal parameter settings for high performance word sense disambiguation. In SENSEVAL-3: Third International Workshop on the Evaluation of Systems for the Semantic Analysis of Text, Barcelona, Spain (2004).

3. Hoste, V., Hendrickx, I., Daelemans, W. and A. van den Bosch. Parameter optimization for machine-learning of word sense disambiguation. Journal of Natural Language Engineering, 8(4) (2002) 311-327.

4. Kilgarriff, A. SENSEVAL: An Exercise in Evaluating Word Sense Disambiguation Programs. In Proceedings of LREC, Granada (1998) 581--588.

5. Lee, Y-K., Ng, H-T., and Chia, T-K. Supervised Word Sense Disambiguation with Support Vector Machines and Multiple Knowledge Sources. In Proceedings of SENSEVAL-3 workshop (2004). 
6. Legrand, S., Pulido JGR. A Hybrid Approach to Word Sense Disambiguation: Neural Clustering with Class Labeling. Knowledge Discovery and Ontologies workshop at $15^{\text {th }}$ European Conference on Machine Learning (ECML) (2004).

7. Luo, F., Khan, L., Bastani F., Yen I-L and Zhou, J. A dynamically growing self-organizing tree (DGSOT) for hierarchical clustering gene expression profiles, Bioinformatics 2004 20(16):2605-2617, Oxford University Press.

8. Manning, C., Tolga Ilhan, H., Kamvar, S., Klein, D. and Toutanova, K. Combining Heterogeneous Classifiers for Word-Sense Disambiguation. Proceedings of SENSEVAL2, Second International Workshop on Evaluating WSD Systems (2001) 87-90.

9. Mihalcea, R. Word sense disambiguation with pattern learning and automatic feature selection. Journal of Natural Language Engineering, 8(4) (2002) 343-359.

10. Mihalcea, R., Kilgarriff, A. and Chklovski, T. The SENSEVAL-3 English lexical sample task. Proceedings of SENSEVAL-3 Workshop at ACL (2004).

11. Montoyo, A. and Suárez, A. The University of Alicante word sense disambiguation system. Proceedings of SENSEVAL-2 Workshop (2001) 131-134.

12. Mooney, R. Comparative experiments on disambiguating word senses: An illustration of the role of bias in machine learning. Proceedings of the Conference on Empirical Methods in Natural Language Processing (1996).

13. Saarikoski, H. mySENSEVAL: Explaining WSD System Performance Using Target Word Features. In NLDB-05, Natural Language for Databases: Proceedings of 10th International Conference on Applications of Natural Language Processing to Information Systems. Lecture Notes in Computer Science, N 3513, Springer (2005) 369-371.

14. Seo, H-C., Rim, H-C. and Kim, S-H. KUNLP system in Senseval-3. Proceedings of SENSEVAL-2 Workshop (2001) 222-225.

15. Strapparava, C., Gliozzo, A., and Giuliano, C. Pattern abstraction and term similarity for Word Sense Disambiguation: IRST at Senseval-3. In Proceedings of SENSEVAL-3 workshop (2004).

16. Witten, I., Frank, E. Data Mining: Practical Machine Learning Tools and Techniques (Second Edition). Morgan Kaufmann (2005).

17. Yarowsky, D., S. Cucerzan, R. Florian, C. Schafer and R. Wicentowski. The Johns Hopkins SENSEVAL2 System Descriptions. Proceedings of SENSEVAL-2 workshop (2002).

18. Yarowsky, D. and Florian, R. Evaluating sense disambiguation across diverse parameter spaces. Journal of Natural Language Engineering, 8(4) (2002) 293-311.

19. Zavrel, J., S. Degroeve, A. Kool, W. Daelemans, K. Jokinen. Diverse Classifiers for NLP Disambiguation Tasks. Comparisons, Optimization, Combination, and Evolution. TWLT 18. Learning to Behave. CEvoLE 2 (2000) 201-221 